\title{
Communication Disconnection Prevention System by Bandwidth Depression-Type Traffic Measurement in a Multi-Robot Environment using an LCX Network
}

\author{
Kei Sawai ${ }^{1}$, Satoshi Aoyama ${ }^{2}$, Takumi Tamamoto ${ }^{3}$, Tatsuo Motoyoshi ${ }^{4}$ \\ Hiroyuki Masuta ${ }^{5}$, Ken'ichi Koyanagi $^{6}$, Toru Oshima ${ }^{7}$ \\ Department of Intelligent Robotics \\ Toyama Prefectural University \\ Toyama, Japan
}

\begin{abstract}
In this paper, we propose and develop a method for determining the transmission amount of each mobile robot connected to a network constructed with a leaky coaxial cable (LCX) by using broadcast packets. Tele-operation of mobile robots using an LCX network is more effective as an information collection method in closed spaces compared with existing methods in terms of the maintenance of the mobile robots' running performance and the stability of the communication quality for disaster reduction activity. However, when the transmission and reception of information exceeds the maximum transmission amount, communication disconnection and transmission amount reduction occur because of band division in the communication path, and there is a risk that mobile robots will be separated from the LCX network. Therefore, to prevent the network division and the decrease of transmission amount during multi-robot operation on an LCX network, we propose a method for determining the transmission amount of each mobile robot using broadcast packets. The proposed method is evaluated on an LCX network, and its effectiveness is confirmed by evaluating the transmittability of broadcast packets and operability of mobile robot.
\end{abstract}

Keywords-Multi-robot; tele-operation; leaky coaxial cable; LCX networks; operability; broadcast packets; transmittability of broadcast packets; network disconnection prevention; disaster reduction activity

\section{INTRODUCTION}

After a disaster occurs, disaster reduction activities are conducted to minimize damages, injuries, and fatalities. The investigation of the Hanshin-Awaji Earthquake indicated that survivors buried in the rubble had a survival rate of less than $5 \%$ after 72 hours. Therefore, the prompt implementation of disaster reduction activities is required to save lives [1-2]. To carry out effective disaster reduction activities, it is necessary to quickly gather information about the affected area for disaster deduction activities [3-4]. Drones and existing sensor systems are often used to gather disaster information. However, in closed spaces such as underground facilities and factories, it is impossible to gather information using drones, and there is a high risk that the existing infrastructure is disconnected from the outside [5-6]. In these situations, information must be collected by rescue teams, but there may be risks of secondary disasters such as fire and collapse. Therefore, a rapid information-gathering system using multiple robots is considered. In a multi-robot system, it is necessary to choose communication methods to use between robots and between robots and operators according to the operation environment.

Wired communication and wireless communication can be selected for the communication of mobile robots operating in the disaster area according to the environmental conditions. Wired communication is excellent for maintaining the communication quality between the remote operator and the mobile robot, but in a multi-robot environment, there is a high risk that cable tangle and disconnection may cause communication failure. Although wireless communication improves the running performance of a mobile robot, it is difficult to maintain the communication quality between the operator and the mobile robot because of the influence of structures and obstacles. Therefore, the wireless tele-operation range of the mobile robot is smaller than that of wired communication.

When tele-operation of a mobile robot is required, the communication method that matches the disaster area must be determined, but it is difficult to estimate the situation in the disaster area in advance. Hence, it is important to carry out various studies on tele-operation methods that can be used in closed spaces for effective disaster mitigation activities. It is important to be able to operate all the robots in a multi-robot system without entanglement or damage of cable to collect information quickly in a closed space affected by a disaster.

In this paper, we first discuss the construction method of a multi-robot environment using a leaky coaxial cable (LCX) network, considering the advantages and disadvantages of wired and wireless communication in the tele-operation of mobile robots. Then, we propose a communication disconnection prevention system to address the communication disconnection problem in the multi-robot environment and confirm the system's effectiveness by conducting evaluation experiments in a real environment. In the experiment, we evaluated the proposed method using a mobile robot, and considered the practicality in the real environment such as the afflicted area. The LCX network was also constructed in a real environment and used to evaluate the proposed method. 


\section{CONSTRUCTION OF A Multi-Robot EnVIRONMENT USING AN LCX NETWORK}

\section{A. Tele-Operation of a Mobile Robot using an Ad-hoc Network}

Many studies on the wireless tele-operation of mobile robots in closed spaces have used the Robot Wireless Sensor Network (RWSN) system, which uses an ad-hoc network as a communication method [7-23]. RWSN is a system in which the mobile robot extends the tele-operation area by deploying a wireless communication repeater on a moving route (Fig. 1). However, when the number of relays on the communication path increases, the throughput value between the operator and the mobile robot decreases. The decrease of the throughput value not only reduces the operability but also increases the communication disconnection risk. Therefore, the operator must constantly monitor the communication quality with the mobile robot. In addition, in a multi-robot environment constructed using RWSN, the routing becomes complicated, so the number of relays is likely to change, thus causing the throughput to change. The fluctuation of the throughput leads to the disconnection of communication; thus, multi-robot operation using RWSN is difficult. In this study, to overcome the shortcomings of RWSN, we develop a tele-operation system for mobile robots using an LCX network as the communication infrastructure for the purpose of effective information-gathering activities in disaster areas.

\section{B. A Multi-Robot System using an LCX Network as Wireless Communication Infrastructure}

An LCX network has a large number of holes called slits in the outer conductor of the coaxial cable that are designed to leak the electrical signal transmitted in the cable to the outside (Fig. 2). Information transmitted by the remote operator to the mobile robot is leaked to the outside as radio waves from the slit of the LCX. Slits can also receive radio waves, enabling mutual communication between the mobile robot and the operator.

Therefore, the LCX can be used as a single antenna by connecting it to a TCP/IP-compliant access point (Fig. 3). By laying LCX, a network can be constructed in a wide area along the cable; thus, LCX can be used as wireless communication infrastructure in a multi-robot environment.

\section{Tele-Operation of Multiple Robots using an LCX Network}

An information-gathering system for multiple robots on an LCX network in a closed space affected by a disaster is illustrated in Fig. 4 [24]. In the flow of information-gathering activities, a cable-laying robot first lays LCX as the communication infrastructure. Multiple robots that gather information are operated by wireless tele-operation without degrading the running performance by using radio waves leaked from the laid LCX.

The communication protocol of this system uses TCP / IP in consideration of mobile robot installation and tele-operation. At present, it is common to use a general-purpose PC for the control system of the mobile robot, and TCP/IP is often used as a communication protocol for tele-operation. Therefore, in the proposed system, we adopted IEEE802.11g as the communication method of the mobile robot, and we performed tele-operation by packet communication.

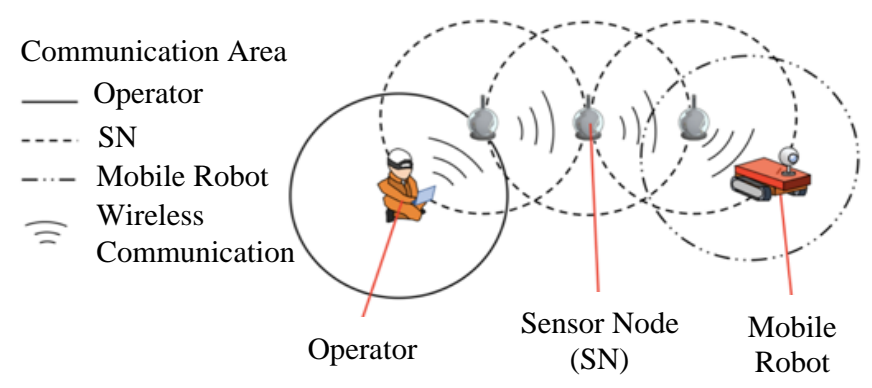

Fig. 1. Robot Wireless Sensor Network (RWSN).

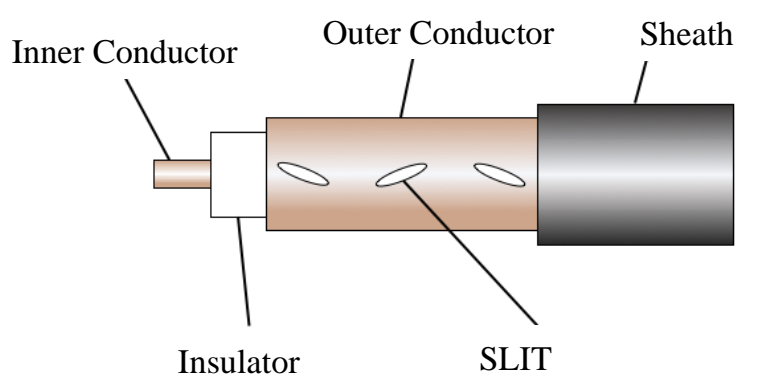

Fig. 2. Internal Structure of the Cable used in an LCX Network.

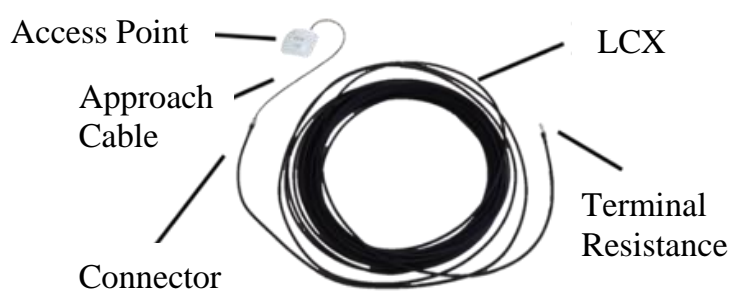

Fig. 3. An LCX Network Equipped with TCP/IP-Compliant Access Points.

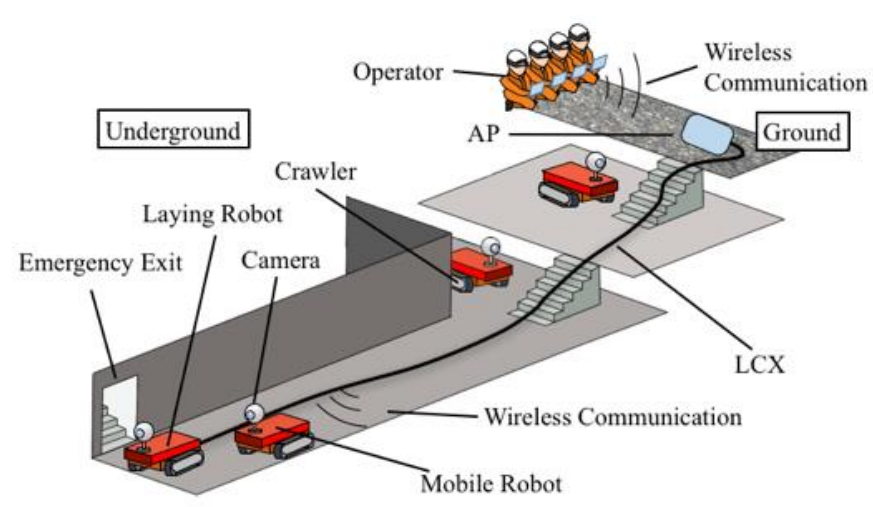

Fig. 4. A Disaster Area Information-Gathering System utilizing LCX Network Construction. 
IEEE 802.11g has a theoretical throughput value of 54.0 Mbps, which is the amount of data that can be transmitted and received per unit time. Nitta et al. are conducting research on a wireless tele-operation method for excavators by streaming camera images from the excavator to the operator, and reports that wireless tele-operation requires a throughput value of 4.0 Mbps or more. Thus, in the proposed system, the throughput value required for tele-operation between the operator and the mobile robot is set to $4.0 \mathrm{Mbps}$. IEEE802.11g satisfies the throughput value required for the transmission and reception of streaming and sensor information for tele-operation and command packets used as operation commands, and it can be used in the communication infrastructure of the proposed system.

\section{Considerations for a Multi-Robot System using an LCX Network}

When an operator remotely controls a mobile robot, it is important for the mobile operator to monitor the communication quality between him/her and the mobile robot to prevent decreases in the operability of the mobile robot and disconnection from the network. The communication range of an LCX network becomes narrower from the access point to the end of LCX because the transmitted and received signals are attenuated by the cable length. The throughput value is also reduced because the transmission loss increases with the distance from the access point as the communication range decreases. Additionally, regarding data transmission and reception, if the communication capacity of the communication path exceeds the upper limit, some mobile robots connected to LCX may be disconnected from the network. This is a specification based on TCP/IP to prevent communication failure on the network when the network is heavily loaded and to keep the connected computers as connected as possible. Therefore, it is also necessary for the operator to monitor traffic on communication paths in LCX networks. As mentioned above, traffic monitoring is required to sequentially determine the position information and transmission capacity of each robot because communication characteristics change with distance. An important part of traffic monitoring is determination of the maximum transmission capacity with respect to the distance from the access point of LCX in consideration of the throughput characteristic of the LCX network.

The maximum amount of transmission is the sum of the amount of information transmitted and received through the LCX network. When multiple robots are connected to the LCX network, the maximum transmission amount is divided among all the mobile robots (Fig. 5). However, when transmission and reception exceeding this maximum transmission amount are performed, the network connection cannot be maintained; therefore, a mobile robot is disconnected from the LCX network.

This problem is due to the TCP / IP protocol, and similar disconnects occur in TCP / IP compliant LCX networks like this system. We constructed an LCX network and connected five pairs of operator computers and mobile robots to perform bandwidth compression experiments on communication paths in a virtual multi-robot tele-operation environment.

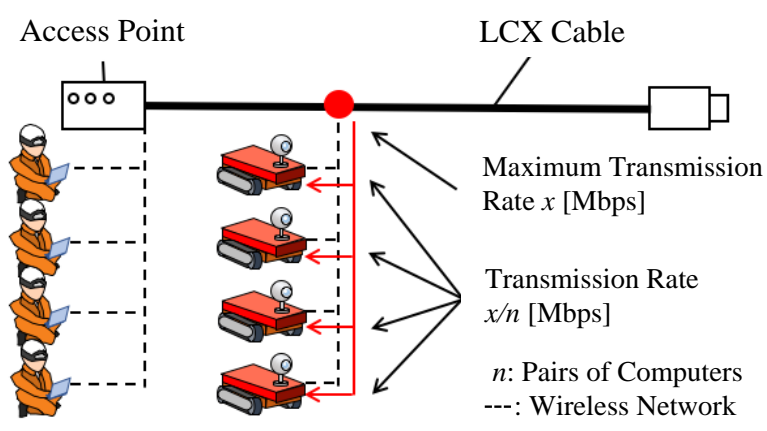

Fig. 5. Fluctuation of Available throughput Value by Band Division in the LCX Network.

In the experiment, the bandwidth division situation was observed with a coiled LCX cable without considering the distance characteristics of the maximum transmission volume (Fig. 6).

The band compression type throughput measurement was performed between all the computers used for tele-operation and the computers assuming mobile robots. The maximum transmission capacity of the LCX network used in the verification experiment was measured to be about $42.0 \mathrm{Mbps}$. As a result of the band compression experiment, the division of the band was confirmed as illustrated in Fig. 7.

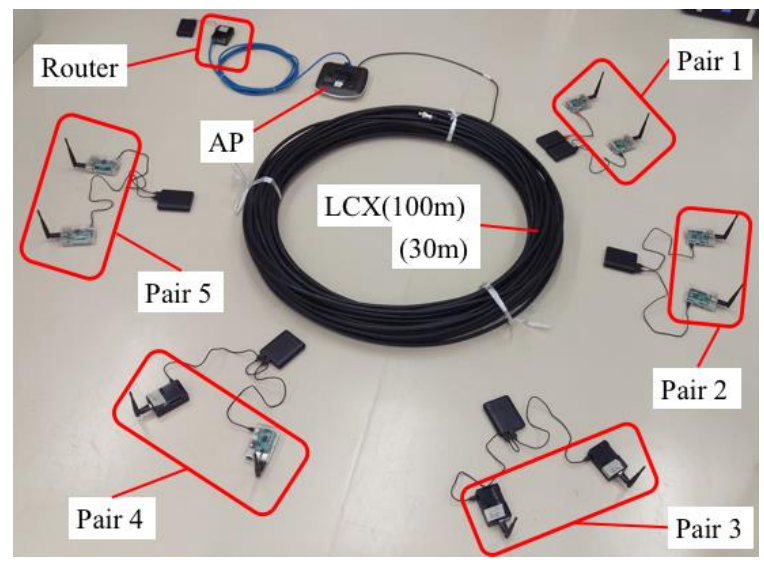

Fig. 6. Observation of a Bandwidth Division Situation in a Coiled LCX Cable without Considering the Distance.

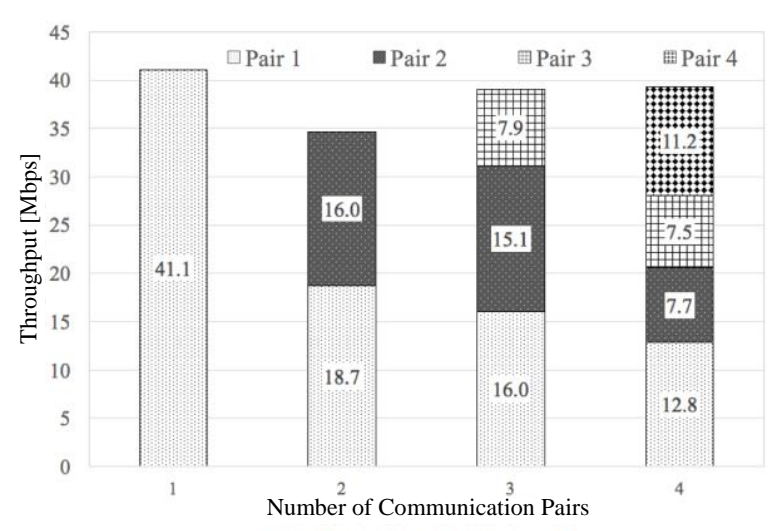

Fig. 7. Throughput Values at Band Division. 
When we used one communication pair, the maximum transmission rate was almost the same as $41.1 \mathrm{Mbps}$. For two or more pairs, band division occurred: for two pairs, the throughput values were 16.0 and $18.7 \mathrm{Mbps}$; for three pairs, the throughput values were 7.9, 15.1, and 16.0 Mbps; and for four pairs, the throughput values were 7.5, 7.7, 11.2, and 12.8 Mbps. However, for five pairs, the connections of all the computers were canceled because the amount of transmission and reception exceeded the maximum capacity. Therefore, for one to execute tele-operation of a multi-robot system using an LCX network, band division must not be generated because it is likely to cause network disconnection. All tele-operators connected to the network must keep track of the amount of transmission and reception between other pairs and be careful not to exceed the maximum amount of transmission. However, in multi-robot operation using an LCX network, no method has been proposed to constantly determine the amount of transmission and reception between other pairs. In a general computer network, bandwidth division control is performed by the resource reservation protocol. However, in the operation of a mobile robot, it is difficult to use the resource reservation protocol because the communication quality changes frequently due to the movement of the robot. Therefore, in this study, we developed a band division prevention method assuming multi-robot operation using an LCX network.

\section{A Traffic Monitoring System using BROAdCast PACKETS THAT STORE TRANSMISSION VOLUME INFORMATION}

This section describes the traffic monitoring system between each tele-operator and a mobile robot, which considers the problems in tele-operation of multiple robots through the LCX network mentioned above. Many methods have been proposed to monitor the communication status on the communication path, but no method has been proposed that considers the operation of the mobile robot. When considering the operation of mobile robots in a disaster area, the type of robot used depends on the type and situation of the disaster. The robots used in the disaster area are not unified, in terms of the structure of the drive system and the type of OS and control system. It is also difficult to estimate the number of mobile robots to be operated. Although many existing communication status monitoring systems are packaged as software, no one software can be introduced to all OSs and communication methods. Additionally, no method has been proposed that uses multiple mobile robots. Thus, in this study, we propose a communication condition monitoring method using broadcast packets on the premise of TCP/IP-based network connection. A broadcast packet-based method can be used freely in TCP/IPcompliant communication, and it can be used regardless of the operating system or communication method. It can also be used regardless of the number of mobile robots connected to the network. Therefore, it is a method that can be implemented in many mobile robot systems.

Fig. 8 presents the flowchart of the proposed system. The tele-operation method prevents band division by determining the maximum transmission value of the communication path and the traffic between other tele-operators and mobile robots and then adjusting the amount of transmission and reception. Additionally, broadcast packets are used for traffic monitoring. Broadcast packets are packets that can be sent to all computers connected to the same network. The mobile robot calculates traffic information from the measurement of transmission and reception information with the tele-operator and then stores the traffic information in a broadcast packet (Fig. 9). For the measurement, we decided to calculate the traffic per unit time. Packets storing traffic information are sent to each computer on the network once per second. Each tele-operator refers to the received broadcast packet and monitors the traffic between other tele-operators and mobile robots sequentially. Then, the tele-operator can prevent communication disconnection due to band division between each mobile robot and the network by controlling the transmission amount before band division occurs. In existing traffic monitoring methods, generally, many methods use bandwidth for traffic measurement packets. In this study, considering the characteristics of the LCX network, we developed a non-bandwidth compression type traffic monitoring system using only broadcast packets.

In the next section, we construct an LCX network that implements the proposed method, and we describe the broadcast transmission and reception experiments.

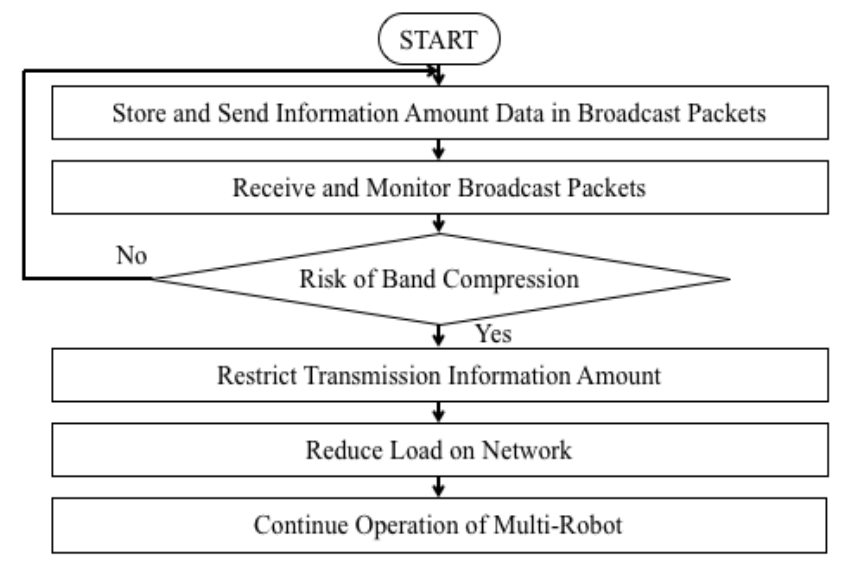

Fig. 8. Flowchart of the Communication Prevention System.

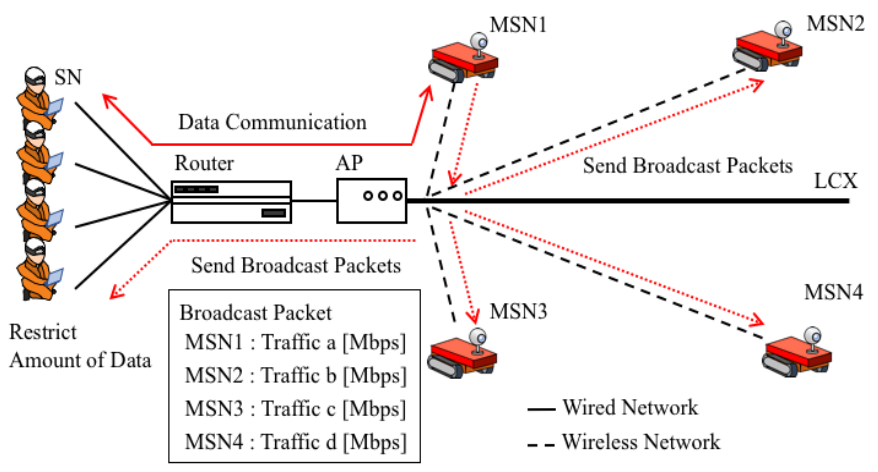

Fig. 9. Proposed Method using Broadcast Packets in a Multi-Robot System. 


\section{EVAluATION EXPERIMENT OF THE TRAFFIC MONITORING SYSTEM USING BROADCAST PACKETS}

\section{A. Evaluation of Packet Loss of the Traffic Monitoring System in an LCX Network using Broadcast Packets}

The purpose of this experiment is to confirm that broadcast packets can be received in an environment where communication load is applied to the communication band. The communication load that can be applied to the communication band is set by assuming the load when information is transmitted and received. In this experiment, we constructed an IEEE 802.11g network using LCX in a real environment and evaluated the availability of the proposed method by transmitting and receiving broadcast packets. The equipment used for the experiment adopted Raspberry Pi2 (Model B) for the control system of the tele-operator and the mobile robot, and LCXF-8D-LCX (HC Networks, Ltd.) for LCX. In addition, AP-214 (Aruba Networks, Ltd.) was used as the access point compliant with TCP/IP. Broadcast packets were sent and received by connecting four tele-operator computers and four computers simulating mobile robots to the LCX network. To put a sufficient communication load on the LCX network, we used four pairs of tele-operators and mobile robots.

\section{B. The Experimental Environment}

Fig. 10 presents the network construction diagram of the network constructed in the experiment. The experimental environment is illustrated in Fig. 11. This experiment was conducted by laying $100 \mathrm{~m}$ of LCX in a straight path where no other wireless LAN existed. The tele-operation computer and the mobile robot were placed at a distance of $0.3 \mathrm{~m}$ in the vertical direction from the LCX. For communication between the mobile robot and the remote operator, UDP protocol was adopted in consideration of the requirement for real-time teleoperation. Tele-operator computers and computers simulating mobile robots were all assigned static IP addresses in consideration of the requirement for using this system in a multi-robot environment. The network configuration in Fig. 3 looks like a bus-type topology, but this network topology was set as star-type in consideration of the ease of connection setup (Fig. 12).

\section{Broadcast Packet Transmission and Reception Experiment}

In this experiment, computers (MSNs 1 to 4) assuming four mobile robots were placed on one mobile robot, and measurement was performed while moving the mobile robots. Tele-operation computers (SN1-4) and the mobile robots (MSN1-4) were placed at $0 \mathrm{~m}$ in measurement environment, and an arbitrary communication load was applied on the communication path between SN1-MSN1 and SN3-MSN3. Next, computers other than SN2 were set to the broadcast packet reception standby mode. In an environment where the communication load is applied to the network, SN2 transmits a broadcast packet that contains information that assumes the amount of data actually transmitted and received 10 times. After that, we checked whether the computers in the reception mode could receive the information stored in the packet. For packet reception confirmation, the number of packet losses was counted as an evaluation of packets that could not be received. The communication loads on the communication path were 5.0,
10.0, 15.0, 20.0, 25.0, and 30.0 Mbps. When the communication load applied to the network was 5.0 to 15.0 Mbps, SN1 generated the communication load. The communication load up to $20.0-30.0 \mathrm{Mbps}$ was generated by $\mathrm{SN} 1$ and SN3. In addition, MSN1-4 was moved from $0 \mathrm{~m}$ to $100 \mathrm{~m}$ at intervals of $10 \mathrm{~m}$, and evaluation experiments were conducted at each $10 \mathrm{~m}$ interval.

\section{Experimental Results}

The experimental results are illustrated in Fig. 13 to 19. The vertical axis represents the number of packet losses, and the horizontal axis represents the distance traveled by each MSN from the access point to the end of LCX. The measurement results for each communication load are presented for each SN and each MSN. From the results of this experiment, we confirmed that the communication load applied to the LCX network is large, and the number of packet losses increases. Additionally, in this experiment, network troubles such as disconnection of communication did not occur at all measurement points, and the mobile robot could be operated in all areas.

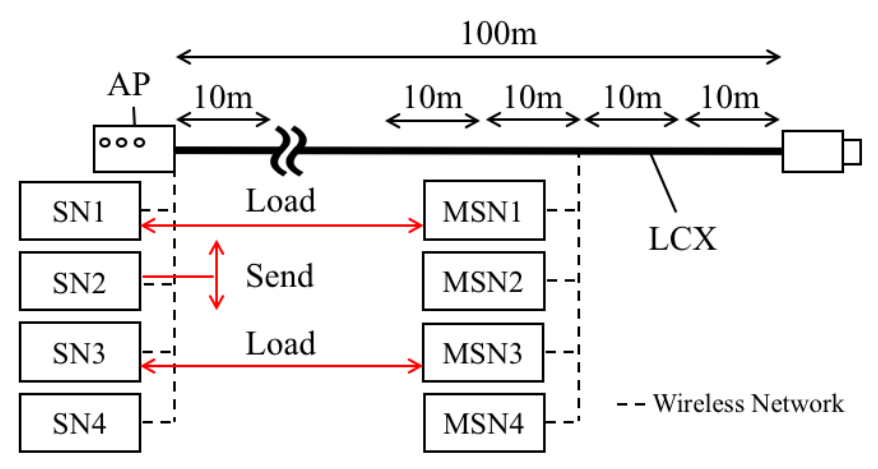

Fig. 10. Network Construction Diagram of Network Constructed in this Experiment.

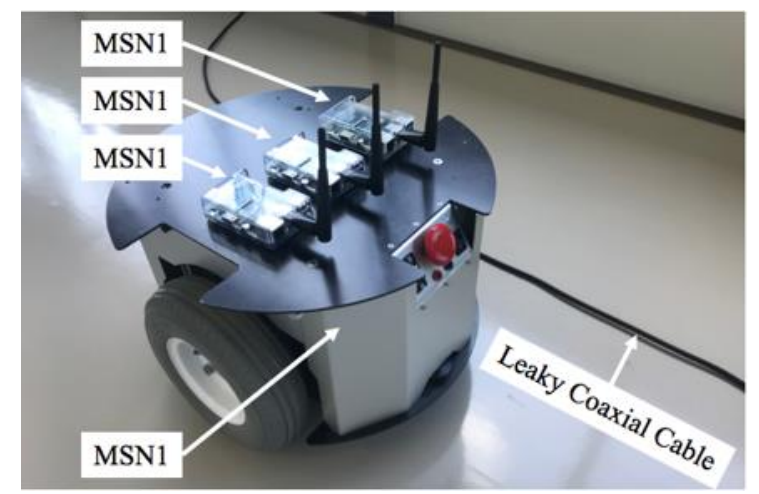

Fig. 11. Experimental Environment of the Proposed Method using Broadcast Packets.

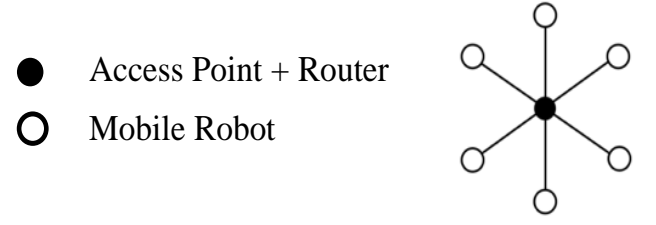

Fig. 12. Wireless Network and Mobile Robot Network Topology Constructed using LCX. 


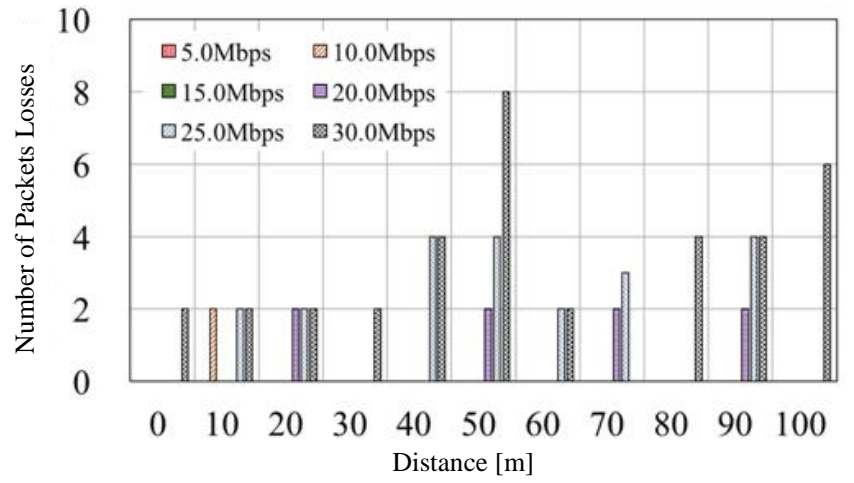

Fig. 13. Number of Broadcast Packet Losses (SN1).

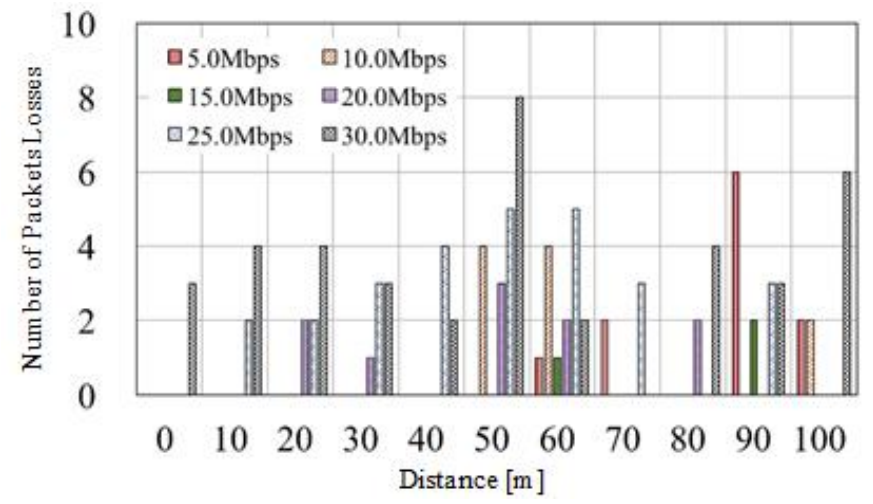

Fig. 14. Number of Broadcast Packet Losses (MSN1).

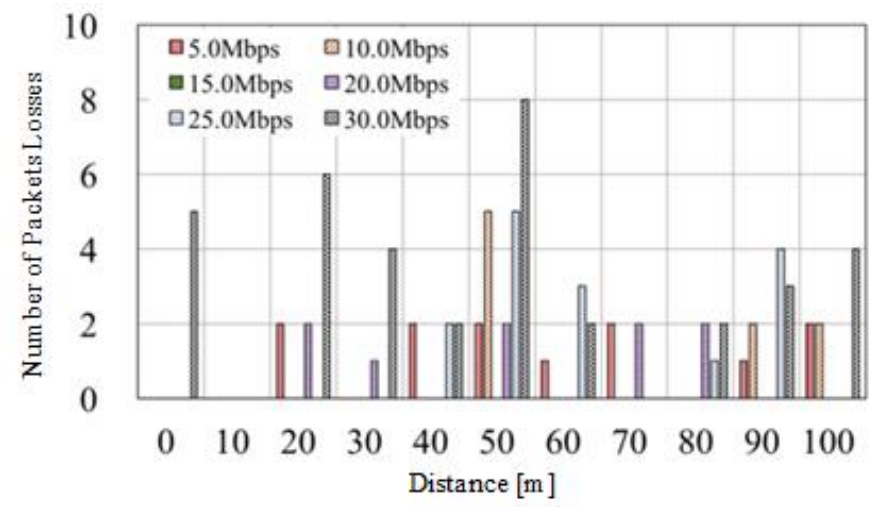

Fig. 15. Number of Broadcast Packet Losses (MSN2).

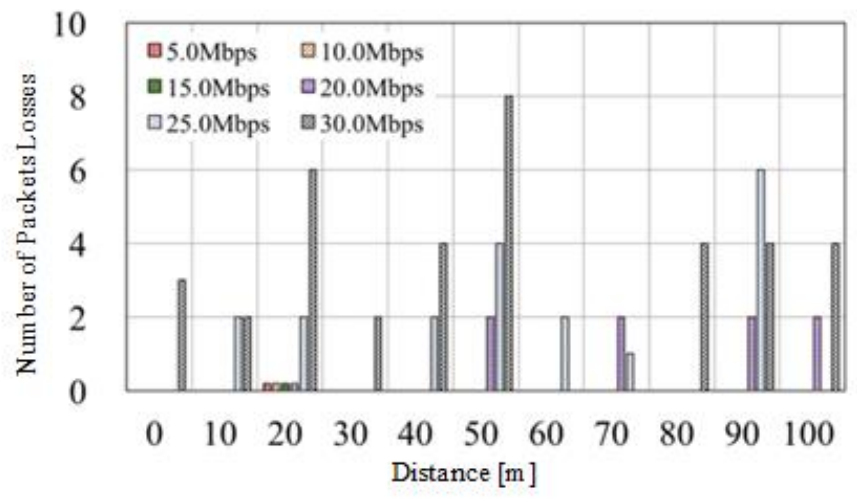

Fig. 16. Number of Broadcast Packet Losses (SN3).

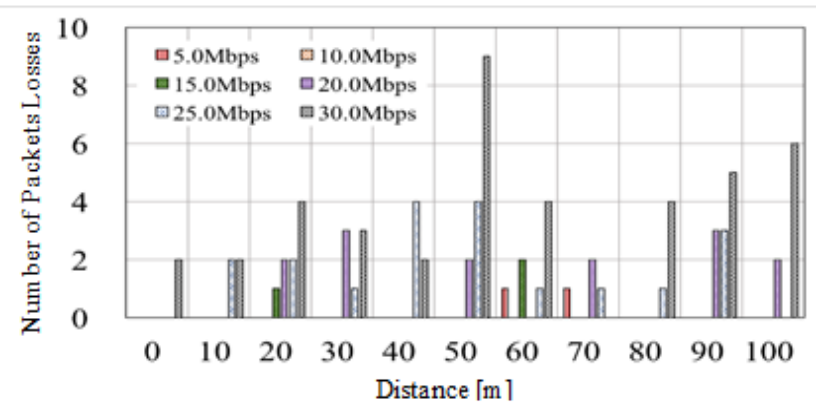

Fig. 17. Number of Broadcast Packet Losses (MSN3).

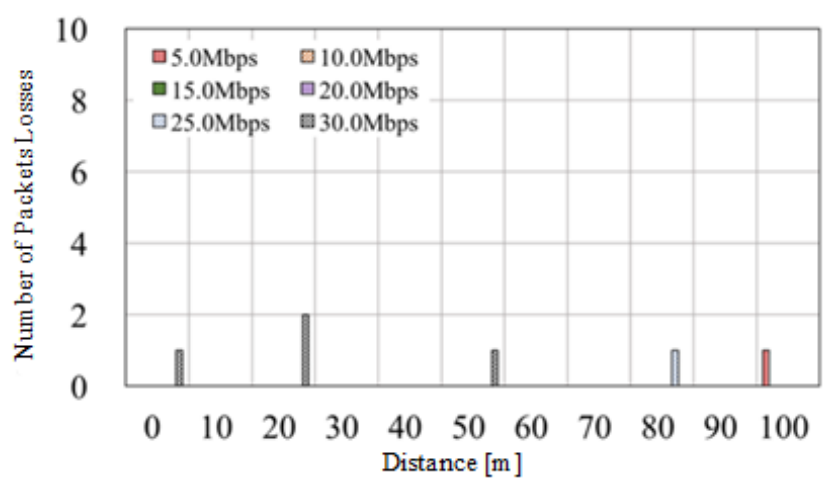

Fig. 18. Number of Broadcast Packet Losses (SN4).

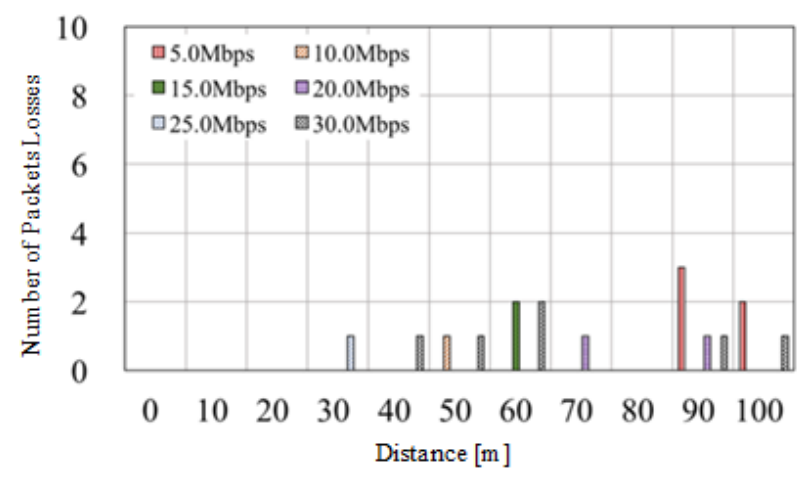

Fig. 19. Number of Broadcast Packet Losses (MSN4).

\section{DISCUSSION}

From the experimental results, it was confirmed that it was possible to determine the communication situation used between each remote operator and a mobile robot connected to the LCX network using the proposed method. The number of packet losses is smaller in each SN than in each MSN. This is thought to be due to the fact that SN2 and other SNs that transmit broadcast packets are placed at the $0 \mathrm{~m}$ point, so the effects of transmission loss of electrical signals in the LCX cable and environmental noise are small. In addition, packet loss was unlikely to occur in each MSN in the range of $0-40 \mathrm{~m}$ while the communication load applied to the communication path was 5.0-15.0 Mbps. This is considered to be due to the fact that the start point of the LCX network was smaller in transmission loss and higher in throughput than the end of the LCX network. Therefore, in the end area of LCX (80-100 m), packet loss occurred even when a communication load of 10.0 15.0 Mbps was applied. Further, as the reason why the number 
of packet losses is not constant, it is conceivable that the communication load changes somewhat due to the change of the communication environment. Furthermore, in this experiment, it was confirmed that broadcast packets were delivered to other computers, although packet loss occurred when the communication load was within $30.0 \mathrm{Mbps}$. Hence, it is considered that the proposed method can prevent the decrease of the transmission amount and communication division due to band division by determining the maximum transmission amount of the communication route to be used. In this experiment, IEEE 802.11g was adopted as the communication method, but even when other communication methods are adopted, this method can be used to determine the maximum transmission amount of the communication path.

\section{CONCLUSION}

In this paper, as a method to prevent disconnection in remote operator-mobile robot communication due to bandwidth compression assuming a multi-robot environment constructed using LCX, a bandwidth non-compression type communication status determining system using broadcast packets was proposed. In LCX cables, the transmission loss of electrical signals increases from the beginning to the end of the cable. The loss of the electrical signal affects the radio waves leaking from the LCX, and the throughput value also decreases. Moreover, when multiple robots connected to LCX are at the same distance from the access point along LCX, the maximum amount of transmission that can be communicated between each remote operator and a mobile robot is divided into bands. The decrease of the throughput value and the bandwidth division reduce the amount of transmission available per mobile robot. As a result, the amount of transmission necessary for tele-operation cannot be secured, and the risk of communication disconnection is increased. In this paper, we proposed a system of preventing communication disconnection and band division by using broadcast packets to obtain information on the transmission amount that each mobile robot connected to LCX transmits and receives for tele-operation. Remote operators can pay attention to communication disconnection and band division because other remote operators can share the amount of transmission that other mobile robots are transmitting and receiving.

For the evaluation experiment of the proposed method, an LCX network was constructed, and its effectiveness was confirmed using a remote operator computer and a mobile robot. The number of broadcast packet losses increased when the communication path was overloaded or when the distance from the access point was increased. If broadcast packets cannot be received, it is not possible to determine the amount of transmission and reception of other mobile robots, so the risk of communication disconnection increases. However, in this experiment, broadcast packets were transmitted 10 times, but in all evaluations, no situation occurred in which packets could not be received. We also consider that the reduction of the number of packet losses can be dealt with by increasing the number of broadcast packet transmissions.

\section{REFERENCES}

[1] CHI Hao-yuan, LIU Xu, XU Xiao-dong: A Framework for Earthquake Disaster Mitigation System, Proceedings of 2011 China located International Conference on Information Systems for Crisis Response and Management (ISCRAM), pp.490-495, 2011.

[2] Y. Kawata: The great Hanshin-Awaji earthquake disaster: damage, social response, and recovery, Journal of Natural Disaster Science, Vol. 17, No. 2, pp.1-12, 1995.

[3] Y. Kawata: Disaster Mitigation due to next Nankai earthquake tsunami occurring in around 2035, Proc. of International Tsunami Symposium 2001, session 1, pp. 315-329, 2001.

[4] Y. Kawata: The great Hanshin-Awaji earthquake disaster: damage, social response, and recovery, Journal of Natural Disaster Science, Vol. 17, No. 2, pp.1-12, 1995.

[5] Abishek T K, Chithra K R and Maneesha V. Ramesh: ADEN: Adaptive Energy Efficient Network of Flying Robots Monitoring over Disaster Hit Area, Proc. of 8th IEEE International Conference on Distributed Computing in Sensor Systems (IEEE DCOSS), pp.306-310, 2012.

[6] Abishek T K, Chithra K R, Maneesha V Ramesh: AER: Adaptive Energy Efficient Routing Protocol For Network of Flying Robots Monitoring over Disaster Hit Area, Proc. of 21st Annual Wireless and Optical Communications Conference (WOCC), pp.166- 169, 2012.

[7] T. Suzuki, K. Sawai, H. Kono and S. Tanabe: Sensor Network Deployment by Dropping and Throwing Sensor Node to Gather Information Underground Spaces in a Post-Disaster Environment, Descrete Event Robot, iConcept PRESS, ISBN 978-1-922227-05-8, 2012, in press.

[8] K. Sawai, H. Kono, S. Tanabe, K. Kawabata, T. Suzuki: Design and Development of Impact Resistance Sensor Node for Launch Deployment into Closed Area, In international journal of sensing for industry(Sensor Review), Emerald Group Publishing Ltd., Vol. 32, pp.318-326, 2012.

[9] S. Tanabe, K. Sawai and T. Suzuki: Sensor Node Deployment Strategy for Maintaining Wireless Sensor Network Communication Connectivity, International Journal of Advanced Computer Science and Applications (IJACSA), The Science and Information organization, Vol.2, No. 12, pp.140-146, 2011.

[10] Y. Koike, K. Sawai and T. Suzuki: A Study of Routing Path Decision Method Using Mobile Robot Based on Distance Between Sensor Node, International Journal of Advanced Research in Artificial Intelligence (IJARAI), Vol. 3, No.3, pp. 25-31, 2014. (http://thesai.org/Publications/ViewPaper?Volume=3\&Issue=3\&Code=IJ ARAI\&SerialNo=5).

[11] K. Sawai, J. Peng and T. Suzuki: Throughput Measurement Method Using Command Packets for Mobile Robot Teleoperation Via a Wireless Sensor Network, International Journal of Advanced Computer Science and Applications (IJACSA), Vol. 7, No. 4, pp. 348-354, 2016. (http://dx.doi.org/10.14569/IJACSA.2016.070446).

[12] T. Michiba, K. Sawai, T. Oshima, K. Koyanagi, H. Masuta, T. Motoyoshi and T. Tamamoto: Development of Communication Quality Measurement Method Utilizing CMD Packet for Mobile Robot Teleoperation", Proc. of the 3rd Fukushima Research Conference on Remote Technologies for Nuclear Facilities 2018, p. 19, 2018.

[13] Y. Ichikawa, K. Sawai, P. Ju and T. Suzuki: Study of Mobile Sensor Node Deployment Method maintaining End to End Throughput in Ad Hoc Networks, Proc. of the 6th International Conference on Advanced Mechatronics (ICAM2015), p. 135, 2015.

[14] K. Sawai, S. Tanabe, R. Kunimoto, Y. Koike and T. Suzuki: Performance Evaluation of Sensor Node Deployment Strategy for Maintaining Wireless Sensor Network Communication Connectivity, Proc. of the 2012 International Symposium on Nonlinear Theory and its Applications (NOLTA2012), pp. 255-258, 2012.

[15] H. Akiyama, K. Yoshida and S. Tadokoro: Field Experiment on Multiple Mobile Robots Conducted in an Underground Mall, Field and Service Robotics Springer Tracts in Advanced Robotics, vol. 62, pp365- 375, 2010. 
[16] H. Jiang, J. Qian, and W. Peng: Energy Efficient Sensor Placement for Tunnel Wireless Sensor Network in Underground Mine, Proc. of 2nd International Conference on Power Electronics and Intelligent Transportation System(PEITS 2009), pp. 219-222, 2009.

[17] J. Xu, S. Duan and M. Li: The Research of New Type Emergency Rescue Communication System in Mine Based on Wi-Fi Technology, Proc. of IEEE 3rd International Conference on Communication Software and Networks (ICCSN), pp. 8-11, 2011.

[18] K. Nagatani, S. Kiribayashi, Y. Okada, K. Otake, K. Yoshida, S. Tadokoro, T. Nishimura, T. Yoshida, E. Koyanagi, M. Fukushima and S. Kawatsuma: Emergency Response to the Nuclear Accident at the Fukushima Daiichi Nuclear Power Plants using Mobile Rescue Robots, Journal of Field Robotics, vol. 30, no. 1, pp. 44-63, 2013.

[19] E. Parker, L. Kannan, B. Xiaoquan, F. Yifan: Heterogeneous Mobile Sensor Net Deployment Using Robot Herding and Line of Sight Formations, Proc. of 2003 IEEE/RSJ International Conference on Intelligent Robots and Systems (IROS2003), Volume 3. pp.2488-2493, 2003.

[20] A. Stoeter, S. Rybski, E. P. Gini, M. Papanikolopoulos : Autonomous stair-hopping with scout robots. Proc. of the IEEE/RSJ Intelligent Robots and Systems, 721-726, 2002.
[21] M. Sugano, T. Kawazoe, Y. Ohta, M. Murata : An indoor localization system using RSSI measurement of a wireless sensor network based on the ZigBee standard. Proceedings of the sixth IASTED International Multi- Conference on Wireless and Optical Communication, 504-508, 2007.

[22] R. Suzuki, K. Makimura, H. Saito, Y. Tobe: Prototype of a sensor network with moving nodes. Special Issue on INSS 2004, Transaction SICE, E-S-1, 52-57, 2006.

[23] R. Suzuki, K. Sezaki, Y. Tobe: A Protocol for policy-based session control in disruption tolerant sensor net- works. Special section on Ubiquitous Sensor Networks, IEICE TRANSACTIONS on Communications, E90-B(12), 3426-3433, 2007.

[24] S. Aoyama, K. Sawai, T. Oshima, K. Koyanagi, T. Motoyoshi, Hiroyuki Masuta and Takumi Tamamoto,: Proposal of Disconnection Prevention System in Multi-Robot System Utilizing LCX Network by Monitoring Broadcast Packets Storing Traffic Information, Proc. of the 3rd Fukushima Research Conference on Remote Technologies for Nuclear Facilities 2018, p. 18, 2018. 